Original Article

\title{
An experiment to classify the clinical fear seen during aerobic movement acquisition in adults with visual impairment
}

\author{
AKIRA Kimura, RPT, $\mathrm{PhD}^{1)}$ \\ 1) Graduate School of Health Sciences, Gunma PAZ College: 1-7-1 Tonyamati, Takasaki, Gunma \\ 370-006, Japan
}

\begin{abstract}
Purpose] This study aimed to classify the fear that occurs during the process of a squat-like movement by persons with visual impairment. [Subjects and Methods] Fifteen persons with a visual impairment, 45 years and older, who were independent in daily living were recruited for the study. The study utilized a field experiment design. The setting was the Kusatsumachi Welfare Center, and the study period was from March through August 2015. Outcome measures included the presence or absence of fear during attempt of a squat-like movement on a force plate. Metrics included the ratio of low and high frequency components of heart rate variability spectra $\left(\mathrm{cm}^{2} /\right.$ $\mathrm{Hz}$ ) based on resting rate intervals (ActiGraph wGT3X-BT, USA). Autonomic hyperactivity was defined as the occurrence of objective fear with LF/HF ratio of over 2.0. Statistics included Fischer's exact test. [Results] Four fear types included the combination of danger and objective fear factors. The frequencies of occurrence of clinically categorized subjective fear across the four fear types were two (danger, fear), seven (no danger, fear), six (danger, no fear), and zero (no danger, no fear) persons. [Conclusion] This procedure of classifying fear might be feasible during evaluation of a squat-like movement by persons with a visual impairment. Key words: Classify, Fear, Visual impairment
\end{abstract}

(This article was submitted Apr. 28, 2016, and was accepted Jul. 7, 2016)

\section{INTRODUCTION}

Approximately $90 \%$ of visually impaired people live in developing countries. About $65 \%$ of all people with a visual impairment are 50 years old and older, and this age group currently comprises about $20 \%$ of the world's population. With an increasing elderly population worldwide, more people will be at risk of visual impairment due to chronic eye diseases and ageing processes ${ }^{1)}$. Serious concern has emerged about the primary medical and nursing care treatment of individuals with disabilities due to their increasing severity and complexity along with their increasing cost to society. In Japan, while the number of patients blinded by diabetic retinopathy is decreasing, the percentage of those receiving dialysis in connection with diabetes increased by $0.7 \%$ from FY2009 to $35.8 \%$ in $2015^{2}$. This converts to 2,320 people per year.

In the rural Japanese community of Kusatsumachi in Gunma prefecture, which was the focus of this study, the rate of dialysis treatment for those who are visually impaired was $3 \%$ out of a total population of $7,120^{3}$. Additionally, the management of lifestyle diseases for those with vision impairment is inadequate. Indeed, compared to sighted people, visually impaired people have limited ability to obtain information necessary to learn about exercise and dietary habits for personal health maintenance. Along with advances in wearable devices, instructional video technology supporting physical activities has been on a rise ${ }^{4}$, and the number of healthy people utilizing it is increasing. However, blind or visually impaired individuals will have inadequate access to such information technology because of the long time it takes to universalize or convert its content to auditory information that would be able to support the physical activities. 
A few studies on physical-activity support for the blind have been published. These studies investigated physical activities in competitive sports through analyzing athletes' movements ${ }^{5)}$ or "clinical fear," a neglected causal factor regarding the training of activities of daily living (ADL) that many people complain of during movement. One possible reason for this is that there are no provisional methods to diagnostically define "clinical fear," and such reports will not lead to the development of evaluative methods to predict performance.

With a focus on recent and interesting theories of cognitive behavioral science, we aimed to develop a clinical diagnostic index as a method to diagnostically define "clinical fear." Additionally, using the presence or absence of a sense of danger as a standard, we examined whether the fear experienced during movement acquisition in adults with visual impairment was classifiable.

Experimenters focused on the presence of an observable "freeze" response, which is believed to be a sign of the participant sensing danger and used as the criterion for determining the presence or absence of a sense of danger. Additionally, "fear" was divided into two categories, fear 1 and fear 2, which from the perspective of early developmental criteria become "innate fear" and "learned fear." From the perspective of full maturity, these could respectively be referred to as"non-dangerous fear," and "dangerous fear." Accordingly, based on whether a corresponding relationship exists between danger and fear, the following four classifications were considered and utilized to classify fear preceding the movement: Type $1=$ danger with fear; Type 2 = danger without fear; Type 3 = no danger with fear; and Type 4 = no danger and no fear. This typological configuration is known as a dual-process model ${ }^{6-8)}$, which has recently been equivalently, expressed, as a 2 -systems model $^{9,10)}$.

The present pilot study aimed to classify the fear that occurs when movement acquisition is impaired based on the interviews and the results of autonomic functional analyses conducted during the process of squat-like movement acquisition undergone for individual health maintenance by adults with visual impairment who had never received aerobic exercise instruction. It further sought to clarify which instructional content was perceived as safe and effective at alleviating fear for participants.

\section{SUBJECTS AND METHODS}

Subjects were fifteen participants, 45 years old and older, selected randomly from a pool of 72 adults with visual impairment who were independently performing activities of daily living (ADL) in Kusatsumachi, Gunma, Japan. The study utilized a field experiment design. The study was conducted at the Kusatsumachi Welfare Center from March through August 2015. Participant recruitment was done by our staff who explained the study intent to the Society for the Visually Impaired (which has approximately 72 members in total) and the Social Welfare Council, both of which were already involved in providing activity support to visually impaired individuals in Kusatsumachi, Gunma Prefecture. Subsequently, we recruited potential research subjects based on verbal communication with relevant parties. Discontinuance criteria specified situations that arise in which there is a legitimate reason for a participant to withdraw from the study (e.g., changes in the home environment, aggravation of disease, declines in physical function).

Ethics review and approval was obtained from the Gunma PAZ College Ethics Committee (No. 15-9). All work was carried out after obtaining informed consent from all participants. Assessments were done in a silent and temperature controlled room without cooling fans. A desk and chair were prepared for observation and listening and a $30 \mathrm{~cm}^{2}$ force plate (Pasco PS2142 force plate, USA) was placed on the floor to measure ground reaction force (GRF). The participants were asked to stand on the plate, and their condition was uncontiuuonsly observed during the daytime from 10 A.M. until 5 P.M. while they performed squat-like movements following verbal instruction.

GRF measurement and the protocol assessed "the level of comprehension of the pointers used in movement execution." In the protocol for movement instruction, the assessor provided verbal instruction to the participant, saying, "To squat, lower your hips towards your feet, bend your knees as far as you can without straining them, and then raise your hips up again." Participants were then prompted with, "Now, to your own ability, let's try a quick and easy set of five repetitions." Participants were then told, "If at any time you feel afraid that you will fall, please call out. I can support you so that you won't fall, but please do your best to complete the exercise by yourself." In provision of verbal support, the assessor also provided feedback when the movement was performed consistently by saying, "You're doing a good job of maintaining your form throughout the repetitions." To assess the comprehension of the pointers used in movement execution, the assessor interviewed the participants and asked about impressions regarding their understanding of movement orientation, the effects of pointers on movement speed, the use of movement repetition timing cues, and pointers regarding spatial location information.

Several outcome measures were measured. Ground reaction force (GRF) was measured on the floor where the participants stood. The presence or absence of subjective fear assessed the presence of anecdotal expressions of fear immediately prior to the squat-like movement on the force plate. Presence or absence of objective fear as thru an anxiety index measures the ratio of low and high frequency components ( $\mathrm{LF} / \mathrm{HF}$ ratio) of heart rate variability spectra $\left(\mathrm{cm}^{2} / \mathrm{Hz}\right)$ based on resting rate (RR) intervals (ActiGraph wGT3X-BT, USA). Autonomic hyperactivity was defined as over 2.0 (LF/HF ratio), indicating that objective fear was occurring.

Statistical analysis first included a typological analysis for which a dual-process theory was adapted to classify clinical fear based on the relationships between danger and subjective and objective fear. The fear types included perception of danger with freeze response that one was in a position to fall from a top a platform, Type 1 is danger with anxiety, Type 2 is 
Table 1. Ground reaction force plate on the floor utilized by participants (persons with visual impairment)

\begin{tabular}{|c|c|c|c|c|c|c|c|}
\hline ID & Gender & $\begin{array}{l}\text { Body height } \\
\text { (cm) }\end{array}$ & $\begin{array}{c}\text { Body } \\
\text { weight }(\mathrm{kg})\end{array}$ & $\begin{array}{l}\text { Vertical } \\
\text { impulse* }\end{array}$ & $\begin{array}{l}\text { Horizontal } \\
\text { impulse* }\end{array}$ & $\begin{array}{l}\text { Mean of } \\
\text { vertical } \\
\text { impulse }\end{array}$ & $\begin{array}{c}\text { Mean of } \\
\text { horizontal } \\
\text { impulse }\end{array}$ \\
\hline 1 & male & 153 & 49.1 & 9,727 & 163 & 1,945 & 33 \\
\hline 2 & male & 157 & 63.4 & 24,096 & 340 & 4,819 & 68 \\
\hline 3 & male & 165 & 57.5 & 18,522 & 326 & 3,704 & 65 \\
\hline 4 & female & 115 & 34 & 18,522 & 326 & 3,704 & 65 \\
\hline 5 & male & 168 & 54.5 & 7,485 & 220 & 1,497 & 44 \\
\hline 6 & male & 149.5 & 49.7 & 29,753 & 109 & 5,951 & 22 \\
\hline 7 & male & 165 & 65.4 & 16,275 & 281 & 3,255 & 56 \\
\hline 8 & female & 141 & 59 & 33,353 & 80 & 6,671 & 16 \\
\hline 9 & male & 161.5 & 54.9 & 22,125 & 151 & 4,425 & 30 \\
\hline 10 & female & 140 & 40.5 & 7,502 & 29 & 1,500 & 6 \\
\hline 11 & male & 169 & 61.6 & 6,816 & 77 & 1,363 & 15 \\
\hline 12 & male & 170 & 66.1 & 8,887 & 152 & 1,777 & 30 \\
\hline 13 & female & 138 & 41.3 & 10,670 & 207 & 2,134 & 41 \\
\hline 14 & male & 167 & 55 & 7,252 & 132 & 1,450 & 26 \\
\hline 15 & female & 142 & 52.9 & 19,443 & 141 & 3,889 & 28 \\
\hline
\end{tabular}

*Impulse: Five repetitions of integrated force $(\mathrm{N} \cdot \mathrm{s})$ of Ground reaction force (GRF), sampling frequency $200 \mathrm{~Hz}$. Pasco 2-Axis Force platform PS2142

Table 2. Cross tabulation of sense of danger with "freeze" and subjective fear in people with visual impairment $(n=15)$ congenital (7) or acquired blindness (8)

\begin{tabular}{lccc}
\hline Acquired or congenital & No fear & Fear & Total \\
\hline Acquired blindness & 3 & 0 & 3 \\
Feeling danger with freeze (n) & 4 & 1 & 5 \\
No feeling of danger with freeze (n) & 7 & 1 & 8 \\
Total & & & \\
Congenital blindness & 3 & 0 & 3 \\
Feeling danger with freeze (n) & 2 & 2 & 4 \\
No feeling of danger with freeze (n) & 5 & 2 & 7 \\
Total
\end{tabular}

A Fischer's exact test in Acquired blindness vs. Congenital blindness by No fear vs Fear: N.S.
Table 3. Cross tabulation of sense of danger with "freeze" and "objective anxiety" as objective

Over or under the set point of LF/HF(2.0) No fear Fear $\quad$ Total

Under

$\begin{array}{lccc}\text { Feeling danger with freeze (n) } & 0 & 1 & 1 \\ \text { No feeling of danger with freeze (n) } & 1 & 0 & 1 \\ \text { Total } & 1 & 1 & 2 \\ \text { Over } & & & \\ \text { Feeling danger with freeze (n) } & 6 & 0 & 6 \\ \text { No feeling of danger with freeze (n) } & 5 & 2 & 7 \\ \text { Total } & 11 & 2 & 13\end{array}$

Fear $(\mathrm{LF} / \mathrm{HF})$ in people with Visual Impairment $(\mathrm{n}=15)$

Fischer's exact test with "Different of set point of LF/HF" by No Fear vs. Fear: N.S.

danger without anxiety, and Type 3 is no danger with anxiety, Type 4 is no fearless. Danger and no anxiety. We then sought the frequency of occurrence of each of these clinical fear types. A Fisher's exact test was conducted to examine the relationships among evaluated fear types and expected types $(\mathrm{p}<0.05)$ using IBM-SPSS ver. 20.

\section{RESULTS}

Participants were asked to stand on a ground reaction force (GRF) plate placed on the floor. Subsequently, they were evaluated on aerobics state, OR their movements were evaluated the presence of an observable "freeze" response (Table 1).

The relationships between a sense of danger and subjective/objective (autonomic arousal) fear during movement were evaluated, and the results are shown in Tables 1 and 2. All 15 participants were able to execute the squat-like movements until the end of the session. The relationships of sensing and not sensing danger with the presence or absence of "freeze" response as optical danger was non-significant. The fear types were able to divide into four types reflecting combinations of danger and objective fear factors. The clinically categorized subjective fear was noted for two (Type $1=$ danger with fear), seven (Type 3 = no danger with fear), six (Type $2=$ danger without fear), and zero (Type $4=$ no danger and no fear) persons for each type (Tables 2 and 3 ). 
Table 4. Cross tabulation of fear and subjective classification in persons with visual impairment $(\mathrm{n}=15)$

\begin{tabular}{|c|c|c|c|c|c|}
\hline Acquired or congenital & & Classification & No fear & Fear & Total \\
\hline \multicolumn{6}{|l|}{ Acquired blindness } \\
\hline \multirow[t]{2}{*}{ Subjective (feeling based) fear } & Feeling danger (n) & Classification 1 & 4 & 1 & 5 \\
\hline & No feeling danger (n) & Classification 2 & 0 & 0 & 0 \\
\hline \multirow[t]{2}{*}{ Subjective (feeling based) no fear } & Feeling danger (n) & Classification 3 & 3 & 0 & 3 \\
\hline & No feeling of danger (n) & Classification 4 & 0 & 0 & 0 \\
\hline Total & & & 7 & 1 & 8 \\
\hline \multicolumn{6}{|l|}{ Congenital blindness } \\
\hline Subjective (feeling based) fear & Feeling danger (n) & Classification 1 & 1 & 1 & 2 \\
\hline Subjective (feeling based) no fear & Feeling danger (n) & Classification 2 & 1 & 1 & 2 \\
\hline Subjective (feeling based) fear & No feeling of danger (n) & Classification 3 & 3 & 0 & 3 \\
\hline Subjective (feeling based) no fear & No feeling of danger (n) & Classification 4 & 0 & 0 & 0 \\
\hline Total & & & 5 & 2 & 7 \\
\hline
\end{tabular}

Fischer's exact test of acquired blindness vs. congenital blindness by classifications: N.S.

Table 5. Cross tabulation of relationship in "acquired or congenital blind, observation fear/no fear, feeling danger, classifications of persons with visual impairment $(\mathrm{n}=15)$

\begin{tabular}{|c|c|c|c|c|c|}
\hline Acquired or congenital & & Classification* & No fear & Fear & Total \\
\hline \multicolumn{6}{|l|}{ Acquired blindness* } \\
\hline \multirow[t]{2}{*}{ Observation (feeling based) fear } & Feeling danger (n) & Classification 1 & 0 & 1 & 1 \\
\hline & No feeling of danger (n) & Classification 2 & 0 & 4 & 4 \\
\hline \multirow[t]{2}{*}{ Observation (feeling based) no fear } & Feeling danger (n) & Classification 3 & 0 & 0 & 0 \\
\hline & No feeling of danger (n) & Classification 4 & 3 & 0 & 3 \\
\hline Total & & & 3 & 5 & 8 \\
\hline \multicolumn{6}{|l|}{ Congenital blindness* } \\
\hline Observation (feeling based) fear & Feeling danger (n) & Classification 1 & 0 & 2 & 2 \\
\hline Observation (feeling based) no fear & Feeling danger (n) & Classification 2 & 0 & 2 & 2 \\
\hline Observation (feeling based) fear & No feeling of danger (n) & Classification 3 & 0 & 0 & 0 \\
\hline Observation (feeling based) no fear & No feeling of danger (n) & Classification 4 & 3 & 0 & 3 \\
\hline Total & & & 3 & 4 & 7 \\
\hline
\end{tabular}

A Fischer's exact test in the matrix cells, acquired or congenital blind vs. classifications: linear-by-linear association, $\mathrm{p}=0.011 ; 1$, linear-by-linear association, $\mathrm{p}=0.019$

The frequencies of occurrence of clinically categorized subjective fear were two, seven, six, and zero persons for types $1,3,2$, and 4 , respectively. When looking at cases in which there was a sense of danger, the relationship of congenital and acquired blindness with the frequency of occurrence of the four fear types was non-significant (Table 4). The clinically categorized frequency of objective fear for those with innate blindness was three, six, zero, and six people in types one, 3, 2, and 4, respectively (Table 5). When looking at cases in which there was a sense of danger, a significant relationship was found between the occurrence frequency of the four fear types and innate people with blindness (Tables 4 and 5).

\section{DISCUSSION}

The findings suggest a possible feasibility of fear classification based on examining the process of a squat-like movement of persons with visual impairment. Our results indicated that a sense of fear and the presence or absence of a sense of danger affected movement learning.

All 15 participants in this study were able to execute the squat-like movements until the end of the experiment. Of these, the LF/HF ratio of HRV was measurable in eight participants until the end of the experiment. When we controlled for objective fear, the relationships among congenital blindness, sense of danger, and fear were significant but when adapting for subjective fear these relationships were non-significant. That our classification method adopted for objective fear (autonomic hyperactivity) found significant relationships independently between congenital blindness, sense of danger, and fear, and did not find significant relationships when adapted to subjective fear, which can be taken as an indication of its effectiveness of optimize subjective fear as follow as Types by " 2 -systems model $l^{9,10) " ~ . ~ I n ~ c o r r o b o r a t i o n ~ w i t h ~ a ~ r e p o r t ~ b y ~ S h i b a s a k i ~ e t ~ a l . ~ i n ~}$ 
which autonomic functional determinants were used in prognosis estimations of elderly persons and exercise function, the circumstances of stable physical function are thought to be predictable and have constant applicability.

Although almost objective fear corresponds to subjective fear, congenitally-blind people do not feel fear when they do not sense danger which may explain why significant independent relationships emerged for clinical fear classifications when autonomic function was included to discriminate objective fear. Innate fear is similar to physiological fear, and it should be manifested in a manner opposite to that of psychological fear.

Thus, the existence of danger, which cannot be learned, may suggest the existence of fear that cannot be verified. If so-called Type 1 was a cognitive process possessed by everyone. This would potentially indicate the existence of Type 2 . The present study confirmed cases that were classifiable as Type 2 fear in those with congenital blindness who sensed neither subjective fear nor danger, a previously unobtainable result in dual-process theory-based classification of fear in sighted people.

It is thought that the present study is the first in the world to successfully verifiably demonstrate the lack of fear manifestation in correspondence with a 2-systems model. Type 2 is re-defined as the predictable "anxiety" that can be described no-orally before exercising in response to an unprecedented event that requires the subject to perform repetitive tasks.

On the other hand, Type 3 is able to defined that cannot be described orally before exercising in response to an unprecedented event that requires the subject to perform repetitive tasks.

Furthermore, through our experiment, we confirmed that Type 2 shows the changes in autonomic nerve functions. Therefore, in reference to the principles of behavioral science, it is possible to express as danger without fear.

Additionally, while Type 1 and Type 2 reflect subjective anxiety, we defined dangerous conditions as those that are evident in subjects before exercising as presence of danger and clearly seen by third parties. When there is clear danger that can be seen objectively, such as the subject's foot being off the base, this is defined as danger. In other cases, it is possible to refer to as no danger.

These operative definitions are possible to adopt to test interactions between fear and danger. Therefore, we defined two types of fear and two types of danger. The motive behind this study is to find a clue to the problem of not being able to acquire certain levels of aerobics activities in a given period compared to able-bodied subjects using these results of oral expression data. This study conducted this research to quantitatively measure the anxiety that goes along with exercise/movements to make circumstantial judgments. The novel finding in the present study identifies shortcomings in our prior understanding of fear response and dual-process models that could not have been explained based on 2 -systems models ${ }^{11)}$. Our new theory hypothesizes that explanations of fear and danger by conventional dual-process models, which do not consider the effects of physical movement, are limited to static conditions and that fear, when accompanied by conditions that demand the acquisition of movement and the mastery of exercise training, is expressed by physiological phenomena ${ }^{12)}$. Thus, even if complaints of subjective fear are not exhibited when providing support for aerobic exercise movement acquisition in adults with visual impairment, an exercise physiologist and physical therapist could give them a better safety in particular conduct by this classification of clinical fear like a human factors. The limitations of this study include the difficulty of statistical estimates due to the small sample size. The present study was limited in terms of insufficient measurement due to the inability to continuously monitor subjective fear over a sufficient length of time. Moreover, our results lack generalizability, as we cannot rule out the possibility of bias based on the unique characteristics of the community examined.

The procedure is used to classify fear might be increased safety feasible during the process of squat-like movement by persons with visual impairment. Unconscious fear does not show to be exist, but the classification of clinical fear based on physiological alterations in autonomic activity could be of significance for the development of instructional methods to support the health of the elderly with visual impairments.

\section{ACKNOWLEDGEMENTS}

We would like to thank the Japanese rural government for its cooperation, along with the Kusatsumachi Visual Impairment Association, the Society for the Physically Disabled, staff at the Kusatsumachi Welfare Center, and all the graduate students at Gunma Paz College who assisted in experimental measurements.

\section{REFERENCES}

1) World Health Organization: Visual impairment and blindness. Fact Sheet No. 282. 2014. Updated August. http://www.who.int/mediacentre/factsheets/fs282/ en/ (Accessed Apr. 10, 2016)

2) Norio H, Shigeru N, Kunitoshi I, et al.: Japanese society for dialysis therapy renal data registry - a window through which we can view the details of Japanese dialysis population. Kidney Int Suppl, 2011, 2015: 15-22.

3) Gunma Prefecture Welfare Association: Statistics of Blindness in Gunma Prefecture Welfare Association Report, 2015 , pp 1-4.

4) Apple Inc: Health Kit. https://developer.apple.com/healthkit/ (Accessed Apr. 10, 2016)

5) Kondo K, Sato N: Motion analysis on crouching start of a track athlete with severe visual impairment. Ann Nihon Univ Sch Dent, $2009,7: 111-115$. 
6) Stanovich KE, West RF: Individual differences in reasoning: implications for the rationality debate? Behav Brain Sci, 2000, 23: 645-665, discussion 665-726. [Medline] [CrossRef]

7) Kahneman D: A perspective on judgment and choice: mapping bounded rationality. Am Psychol, 2003, 58: 697-720. [Medline] [CrossRef]

8) Kahneman D: Thinking, fast and slow, 1st ed. New York: Farrar, Straus and Giroux, 2011, pp 12-115.

9) Strack F, Deutsch R: Reflective and impulsive determinants of social behavior. Pers Soc Psychol Rev, 2004, 8: 220-247. [Medline] [CrossRef]

10) Paivio A: Mind and its evolution: a dual coding theoretical approach. Psychol Rec, 2008, 59: 295-300.

11) Evans JS: Dual-processing accounts of reasoning, judgment, and social cognition. Annu Rev Psychol, 2008, 59: 255-278. [Medline] [CrossRef]

12) Shibasaki K, Ogawa S, Yamada S, et al.: Favorable effect of sympathetic nervous activity on rehabilitation outcomes in frail elderly. J Am Med Dir Assoc, 2015, 16: 799.e7-799.e12. [Medline] [CrossRef] 